CLINICAL STUDY

\title{
Treatment with GH receptor antagonist in acromegaly: effect on cardiac arrhythmias
}

\author{
Renata S Auriemma ${ }^{1}$, Rosario Pivonello ${ }^{1}$, Maria Cristina De Martino ${ }^{1}$, Giuseppe Cudemo ${ }^{2}$, Ludovica F S Grasso ${ }^{1}$, \\ Mariano Galdiero $^{1}$, Ylenia Perone ${ }^{1}$ and Annamaria Colao ${ }^{1}$ \\ Departments of ${ }^{1}$ Molecular and Clinical Endocrinology and Oncology and ${ }^{2}$ Clinical and Experimental Medicine, University 'Federico II', via S. Pansini 5 , \\ 80131 Naples, Italy \\ (Correspondence should be addressed to A Colao; Email: colao@unina.it)
}

\begin{abstract}
Objective: To evaluate the effects of short- and long-term treatment with pegvisomant (PEG) on arrhythmias in acromegalic patients resistant to long-term, high-dose therapy with somatostatin analogs (SA).

Materials and methods: Thirteen patients entered the study. All patients started PEG at initial dose of $10 \mathrm{mg}$ daily and then titrated to $5 \mathrm{mg}$ every 6 weeks on the basis of IGF1. A standard 24-h electrocardiography registration was performed in all patients at baseline and after 6 and 18 months of PEG to evaluate: mean (HR), maximum (MHR), and minimum (mHR) heart rate; pauses number (P) and duration (PD); supraventricular episodes (SEs) number and duration (SED); and ventricular ectopic beats (EB) number and duration (EBD). Left ventricular mass (LVM) was also evaluated by standard echocardiography. Results: A slight but not significant decrease in HR, MHR, and mHR was observed after 6-month PEG, whereas a significant decrease in HR $(P=0.03)$, MHR $(P=0.05)$, and mHR $(P=0.05)$ was found after 18-month PEG compared with baseline. LVM significantly $(P=0.05)$ correlated with MRH $(r=-0.50)$ after short-term treatment, and with HR $(r=-0.54)$ and $\mathrm{mHR}(r=-0.55)$ after long-term treatment. Long-term PEG induced the complete recovery of arrhythmias recorded at baseline in one patient and the improvement of rhythm disorders developed after 6-month therapy in another patient. The prevalence of conduction disturbances passed from 15 to $7.7 \%$ after long-term PEG.

Conclusions: Long-term treatment with PEG reduces HR, MHR, and mHR and improves rhythm abnormalities in acromegaly.
\end{abstract}

European Journal of Endocrinology 168 15-22

\section{Introduction}

Acromegaly is associated with an increased morbidity and mortality for cardiovascular disease, including an increased prevalence of arrhythmias $(1,2)$. The exposure to GH and IGF1 excess induces a typical cardiomyopathy (2), which has been claimed as the most important complication as well as cause of death in acromegaly $(2,3,4,5,6,7,8)$. Acromegalic cardiomyopathy develops precociously and progressively induces an initial cardiac hypertrophy associated with an increased heart rate and cardiac output, altogether configuring the hyperkinetic syndrome (8). In the middle phase, hypertrophy becomes more evident, signs of diastolic dysfunction appear, and insufficient systolic function on effort can be documented. In the end stage of untreated disease, cardiac abnormalities may include systolic dysfunction at rest and heart failure (2).

Cardiac arrhythmias, such as supraventricular and ventricular ectopic beats (EB), paroxysmal supraventricular tachycardia, paroxysmal atrial fibrillation, sick sinus syndrome, and bundle branch blocks, have been recorded in 41-48\% of acromegalic patients, particularly during physical exercise when compared with healthy control subjects $(2,9,10,11,12)$, and, disappointingly, recovery from acromegaly does not seem to significantly improve this rate (10). Recently, it has been demonstrated that the relative risk to develop cardiac arrhythmias is about five times higher in acromegalic patients than in healthy control subjects (11). In particular, the prevalence and the severity of ventricular arrhythmias have been reported to be significantly increased in acromegalic patients (9). Complex ventricular arrhythmias have been observed in $48 \%$ of acromegalic patients compared with $12 \%$ of controls (9), and the rate of ventricular premature complexes has been reported to be strongly related to disease duration and, interestingly, to left ventricular mass (LVM), but not to circulating hormone concentrations (9). However, supraventricular premature complexes do not occur more frequently in acromegalic patients than in the general population (12). Furthermore, acromegalic patients frequently show an 
abnormally prolonged QT interval, configuring the long QT syndrome (13), known as a risk factor predisposing to potentially fatal arrhythmias and sudden cardiac death (14).

Both standard electrocardiography (ECG) and 24-h Holter ECG have clearly documented conduction disorders in acromegaly. However, the standard ECG was able to demonstrate only a minority of rhythm disturbances whereas the Holter ECG was able to also show subclinical conduction disturbances in patients with acromegaly (10).

Control of GH and IGF1 excess, either secondary to surgery or to medical therapy with somatostatin analogs (SA), has been reported to improve or at least to arrest the progression of acromegalic cardiomyopathy. Particularly, 12-month therapy with SA has been reported to successfully improve cardiovascular parameters and cardiomyopathy, leading to a rapid reduction of cardiac hypertrophy and to the improvement of systolic and diastolic performance $(15,16,17)$. First-line SA treatment has also been found to reduce heart rate more significantly than surgery, although the prevalence of arrhythmias was slightly but not significantly changed by both treatments (18). In acromegalic patients resistant to treatment with SA, the $\mathrm{GH}$ receptor antagonist pegvisomant (PEG) has been reported to significantly reduce cardiac mass and to increase ejection fraction in order to improve cardiac structure and performance (19). To the best of our knowledge, no data are available today on the effects of PEG on rhythm abnormalities in acromegaly.

The present, open-label, prospective study is aimed at investigating the effects of short-term (6 months) and long-term (18 months) treatment with PEG on rhythm disturbances in a cohort of acromegalic patients proven to be resistant to long-term high-dose treatment with SA.

\section{Materials and methods}

At study entry, all patients provided a written informed consent, and the Ethics Committee of the University 'Federico II' of Naples approved the study. The inclusion and exclusion criteria, patient characteristics, hormonal assays, and treatment protocol have been described in two previous papers $(19,20)$ in which clinical, biochemical, and radiological parameters as well as cardiac structure and performance were considered, but cardiac rhythm abnormalities were not evaluated.

\section{Patients}

Nineteen patients (eight males and 11 females, aged $47 \pm 11$ years) were enrolled in this study. All patients but three had previously undergone neurosurgery and three had also received radiotherapy after unsuccessful surgery. All patients but three had been previously treated with SA (octreotide LAR $40 \mathrm{mg}$ monthly or lanreotide $120 \mathrm{mg}$ monthly) for at least 6 months before study entry, without achieving clinical and biochemical control of acromegaly. In fact, in our patients, SA induced a reduction ranging from 8 to $36 \%$ in IGF1 levels, so patients were considered clearly resistant to SA, although they could be defined totally resistant in some cases and partially resistant in many cases. Among patients who did not receive treatment with SA before study entry, one entered the study immediately after diagnosis, whereas two received presurgical SA and had disease recurrence after an apparently successful surgery performed more than 10 years before. During the study, six patients prematurely dropped out due to poor compliance with the study drug in four patients after 6 months, acute and severe increase in liver enzymes in one patient after 6 months, and progressive increase in tumor size in one patient after 1 year. Among patients who prematurely discontinued PEG, the man treated with $\beta$-blockers was included. Thus, 13 patients (four males and nine females, aged $44 \pm 9$ years) completed the study. Among patients, none showed abnormalities in serum calcium and/or potassium levels, and none had subclinical or overt hyperthyroidism. The patient profile at study entry is shown in Table 1.

\section{Study design}

This study is an open-label, prospective study. After the baseline evaluation, all patients started the 18-month therapy with PEG. Clinical parameters, including height, weight, BMI, and hemodynamic parameters, including heart rate (HR), systolic (SBP), and diastolic (DBP) blood pressure, were recorded at study entry and every 6 weeks thereafter. Similarly, biochemical parameters (fasting glucose and insulin, serum triglycerides, and cholesterol) and safety parameters (liver enzymes) were measured at baseline and every 6 weeks thereafter. LVM was also evaluated by standard echocardiographic method. This study considered three points: the baseline evaluation, the short-term (6 months) evaluation, and the long-term (18 months) evaluation.

Table 1 Patient profile at study entry.

\begin{tabular}{ll}
\hline Number of patients & 13 \\
Age (years) & $44 \pm 9$ \\
BMI $\left(\mathrm{kg} / \mathrm{m}^{2}\right)$ & $28.9 \pm 4.3$ \\
Disease duration (years) & $11.5 \pm 5.4$ \\
GH $(\mu \mathrm{g} / \mathrm{l})$ & $29.9 \pm 40.1$ \\
IGF1 $(\mu \mathrm{g} / \mathrm{l})$ & $719 \pm 156$ \\
ULN & $2.9 \pm 0.7$ \\
Glucose $(\mathrm{mg} / \mathrm{dl})$ & $100 \pm 23.5$ \\
Systolic blood pressure $(\mathrm{mmHg})$ & $130 \pm 15$ \\
Diastolic blood pressure $(\mathrm{mmHg})$ & $86.5 \pm 13$ \\
LVM $(\mathrm{g})$ & $221 \pm 61.5$ \\
HR $(\mathrm{bpm})$ & $73 \pm 6.7$
\end{tabular}

LVM, left ventricular mass; HR, heart rate. 


\section{4-h Holter ECG study}

A standard 24-h Holter ECG recording was performed in all patients to investigate mean (HR), minimum (mHR), and maximum heart rate (MHR) and to detect number $(\mathrm{N})$ and duration (D) of arrhythmias, such as sinus pauses $(\mathrm{P})$, supraventricular episodes $(\mathrm{SE})$, and ventricular EB, at baseline and after 6 and 18 months of treatment with PEG. The 24-h ECG was recorded on digital flash memories and then analyzed by a specific software in order to measure all QRS complexes as well as HR, mHR, and MHR and also to identify occasional arrhythmias that could not be revealed by a standard ECG strip. Patients were asked to record a personal diary of all the activities played during the whole monitoring. Pathological supraventricular and/or ventricular arrhythmias were diagnosed when the number of SE and/or ventricular premature beats exceeded 50/24 h.

\section{Statistical analysis}

Data were analyzed using SPSS Software for Windows, version 15.0 (SPSS, Inc., Cary, NC, USA package). Data are reported as mean \pm s.D. unless otherwise specified. The effects of PEG treatment were analyzed by nonparametric test using Wilcoxon test. The comparison between the prevalence of rhythm abnormalities before and after treatment was performed by $\chi^{2}$ test corrected by Fisher exact test if necessary. The correlation study was performed by linear regression analysis calculating the Pearson's coefficient. Significance was set at $5 \%$.

\section{Results}

\section{Baseline}

After a 4-month washout of long-acting SA, as per protocol, all patients had IGF1 levels at least 1.3 times above the upper limit of normality. LVM, calculated by the Devereux's equation (21), was above the normal range in both men $(241 \pm 24 \mathrm{~g}$, cutoff point $>177 \mathrm{~g})$ and women $(192 \pm 52 \mathrm{~g}$, cutoff point $>118 \mathrm{~g})$. The 24-h ECG monitoring revealed no conduction abnormalities in $85 \%$ of patients. Rhythm disorders, including overt sinus tachycardia and SEs, were found in 15\% of patients. Particularly, a 55-year-old woman showed 36 asymptomatic, nocturnal Ps with PD $<2.5$ s, six SEs with SED of $400 \mathrm{~ms}$, and one EB with EBD of $200 \mathrm{~ms}$. A 47-year-old woman had sinus tachycardia, with HR being 102 bpm at rest.

\section{Short-term (6 months) treatment with PEG}

At a mean dose of $20.8 \pm 5.3 \mathrm{mg}$ and a median dose of $25 \mathrm{mg}$ daily of PEG, IGF1 significantly decreased $(P=0.001)$ compared with baseline and resulted in fully normalized levels in $65 \%$ of patients. GH levels were only slightly but not significantly reduced. The change in GH and IGF1 levels and the concomitant changes in the metabolic profile after short-term treatment with PEG are shown in Table 2. No significant change was found in $\mathrm{HR}, \mathrm{mHR}$, and MHR (Fig. 1), as well as in number and duration of $\mathrm{P}, \mathrm{SE}$, and $\mathrm{EB}$. However, in the youngest patient, who showed no rhythm abnormality at baseline, 21 SEs with SED of 920 ms were recorded after 6-month PEG (Fig. 2).

\section{Long-term (18 months) treatment with PEG}

At mean dose of $25.4 \pm 10.5 \mathrm{mg}$ and a median dose of $25 \mathrm{mg}$ daily of PEG, IGF1 was significantly decreased $(P=0.001)$ compared with baseline, whereas no further reduction was found compared with 6-month evaluation. IGF1 levels were fully normalized in $85 \%$ of patients. $\mathrm{GH}$ levels were similar to those recorded at baseline evaluation and only slightly, although not significantly, increased compared with short-term study. The change in GH and IGF1 levels and the concomitant

Table 2 Effects of short- (6 months) and long (18 months)-term treatment with pegvisomant on clinical, biochemical, and hemodynamic parameters and on heart rate.

\begin{tabular}{|c|c|c|c|c|c|c|}
\hline & Baseline (A) & 6 Months $(B)$ & 18 Months $(C)$ & $A$ vs $B$ & $A$ vs $C$ & B vs C \\
\hline IGF1 $(\mu \mathrm{g} / \mathrm{I})$ & $719 \pm 156$ & $280 \pm 186$ & $279 \pm 230$ & 0.001 & 0.001 & NS \\
\hline $\mathrm{GH}(\mu \mathrm{g} / \mathrm{l})$ & $29.8 \pm 40$ & $25.2 \pm 31.6$ & $30.3 \pm 41.8$ & NS & NS & NS \\
\hline BMI $\left(\mathrm{kg} / \mathrm{m}^{2}\right)$ & $28.9 \pm 4.2$ & $29.2 \pm 4.5$ & $28.6 \pm 3.0$ & NS & NS & NS \\
\hline $\mathrm{SBP}(\mathrm{mmHg})$ & $130 \pm 15.3$ & $128.1 \pm 12.3$ & $126.1 \pm 22.2$ & NS & NS & NS \\
\hline DBP (mmHg) & $86.5 \pm 13.1$ & $84.6 \pm 9.5$ & $80 \pm 12.1$ & NS & NS & NS \\
\hline LVM (g) & $220.9 \pm 61.5$ & $213.3 \pm 59.2$ & $200.3 \pm 51.9$ & 0.03 & 0.006 & 0.02 \\
\hline HbA1c (\%) & $5.4 \pm 0.6$ & $5.1 \pm 0.4$ & $4.9 \pm 0.3$ & NS & 0.05 & 0.05 \\
\hline Insulin ( $\mu \mathrm{U} / \mathrm{ml})$ & $12.6 \pm 6.9$ & $12.5 \pm 6.1$ & $9.4 \pm 5.3$ & NS & NS & NS \\
\hline Triglycerides (mg/dl) & $118.5 \pm 43.9$ & $115.5 \pm 66.7$ & $120.9 \pm 45.8$ & NS & NS & NS \\
\hline Cholesterol (mg/dl) & $219.8 \pm 45.7$ & $218.5 \pm 31.5$ & $221.7 \pm 35.9$ & NS & NS & NS \\
\hline $\mathrm{HR}(\mathrm{bpm})$ & $82 \pm 5.7$ & $78.8 \pm 7.02$ & $76.1 \pm 8.5$ & NS & 0.03 & NS \\
\hline mHR (bpm) & $57.7 \pm 8.7$ & $55.4 \pm 7.3$ & $50.6 \pm 10.8$ & NS & 0.05 & 0.02 \\
\hline MHR (bpm) & $128.1 \pm 12.1$ & $126.6 \pm 11.2$ & $124.3 \pm 14.1$ & NS & 0.05 & NS \\
\hline
\end{tabular}

$\mathrm{HR}$, mean heart rate; $\mathrm{mHR}$, minimum heart rate; $\mathrm{MRH}$, maximum heart rate. 

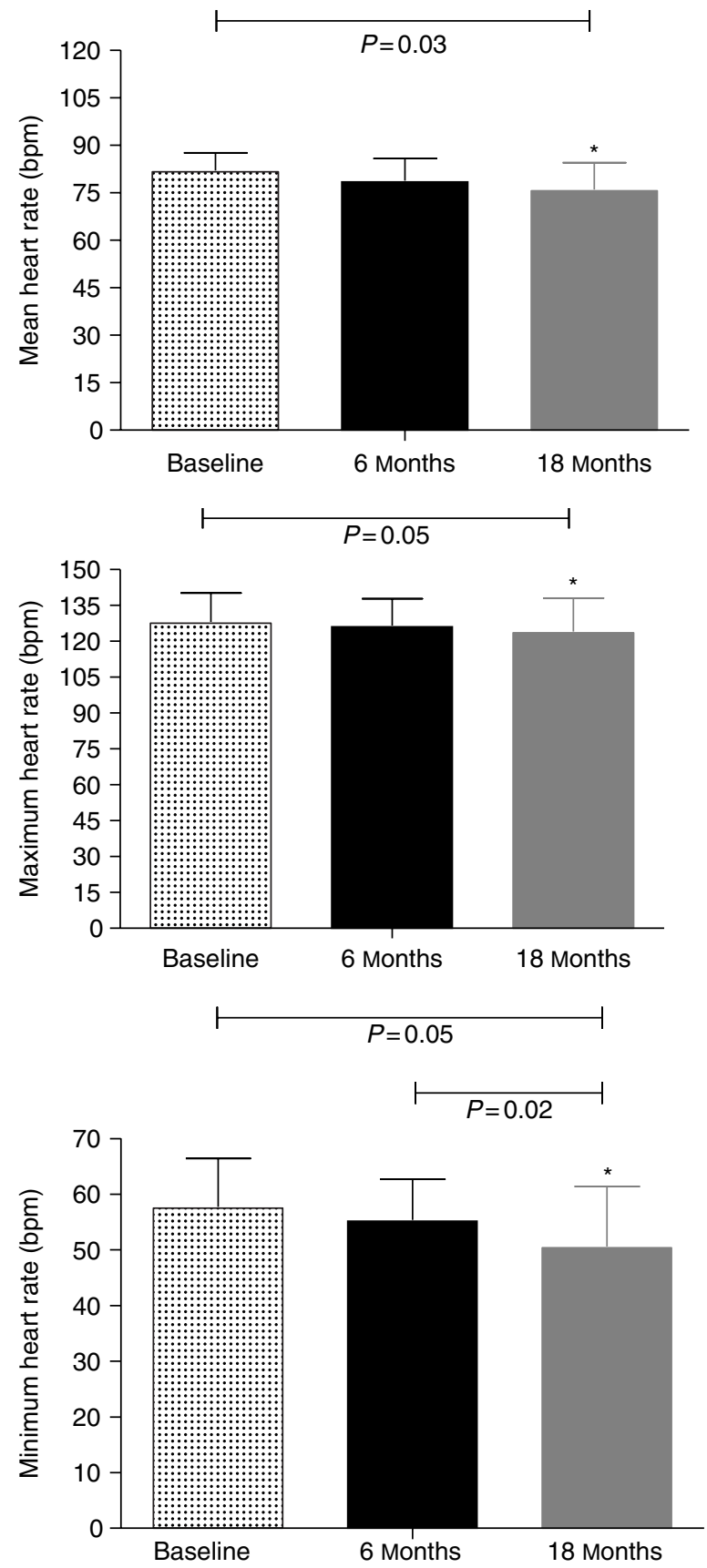

Figure 1 Effects of short- (6 months) and long (18 months)-term treatment with pegvisomant on mean heart rate (top panel), maximum heart rate (centre), and minimum heart rate (bottom). * Mean heart rate $(P=0.03)$, minimum heart rate $(P=0.05)$ and maximum heart rate $(P=0.05)$ were signigificantly decreased compared with Baseline and minimum heart rate was further reduced $(p=0.02)$ compared with 6-month therapy.

change in the metabolic profile after long-term treatment are shown in Table 2. LVM was significantly lower $(P=0.006)$ than baseline and further reduced $(P=0.02)$ compared with short-term study. HR $(P=0.03)$, mHR $(P=0.05)$, and MHR $(P=0.05)$ were significantly decreased when compared with baseline evaluation, and $\mathrm{mHR}$ was further reduced $(P=0.02)$ compared with short-term study (Fig. 1). After 18 months of treatment, in the youngest patient, SEs spontaneously decreased in terms of number (2) and duration $(600 \mathrm{~ms})$, although four asymptomatic, nocturnal Ps with PD $<2.5$ s were recorded, compared with 6-month therapy (Fig. 2). Conversely, in the 55-year-old woman with overt conduction abnormalities at baseline, the 24-h ECG monitoring showed the complete disappearance of abnormal P, SE, and EB after long-term PEG treatment (Fig. 2). Similarly, in the 47-year-old woman with sinus tachycardia at baseline, the reduction of $\mathrm{HR}$ was recorded after long-term therapy with PEG $(\mathrm{HR}=82 \mathrm{bpm}$ at rest). After longterm therapy with PEG, prevalence of rhythm abnormalities passed from 15 to $7.7 \%$.

\section{Correlation study}

The results of the correlation study are shown in Fig. 3. At short-term evaluation, LVM significantly correlated with MHR $(r=-0.55, P=0.05)$ but not with IGF1, $\mathrm{HR}$, and mHR. Percent decrease in LVM $(\Delta \mathrm{LVM})$ did not correlate with percent decrease in IGF1 ( $\Delta \mathrm{IGF} 1)$ and cardiac rhythm parameters. At long-term evaluation, LVM significantly correlated with HR $(r=-0.54$, $P=0.05)$ and mHR $(r=-0.55, P=0.05)$ but not with MHR, and $\triangle \mathrm{LVM}$ significantly correlated with $\Delta \mathrm{IGF} 1$ $(r=0.54, P=0.05)$ and with $\Delta \mathrm{MHR}(r=0.53, P=0.05)$.

\section{Discussion}

This prospective study first demonstrated that treatment with PEG is not arrhythmogenic and induces a significant decrease in heart rate as recorded by the 24-h Holter ECG monitoring in acromegalic patients. Conduction disorders have been recorded in $41-48 \%$ of patients with active acromegaly $(9,10,11,12)$, even in the earlier phase of acromegalic cardiomyopathy (8), and in around $17 \%$ of patients with disease remission after 6-month lanreotide (22). In the majority of cases, rhythm abnormalities at Holter ECG have been described as complex ventricular arrhythmias (9, 10, 12). In the series of active patients of the current study, a lower prevalence of rhythm abnormalities (15\%), mainly including SEs, was found compared with previous literature. It is noteworthy that the great majority of patients of the current study had a long history of the disease and most patients were proven to be resistant to high-dose treatment with SA, which was performed for a long period, although with suboptimal response. The reason for a different prevalence and type of cardiac rhythm disorders in the series of patients in the current study is not known. However, the possibility that this evidence is directly related to beneficial effects of previous treatment with SA on 
Case no.1
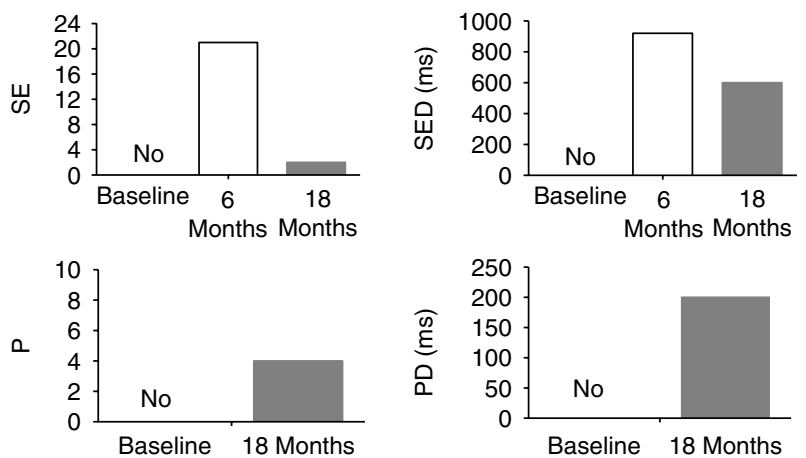

Case no.2
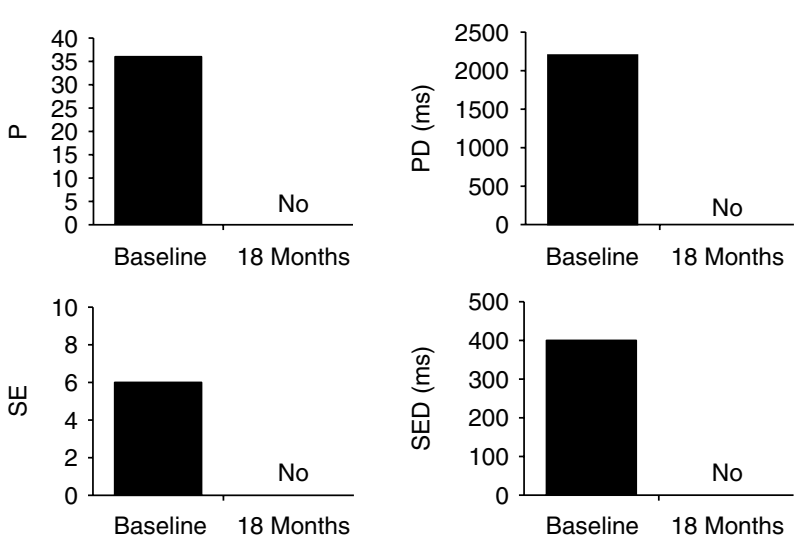
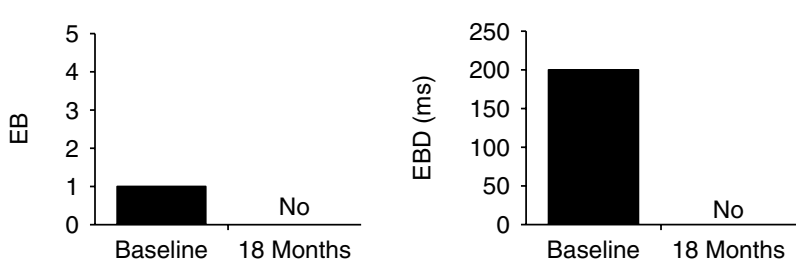

Figure 2 Top panels, case no. 1. The youngest patient, who did not show rhythm disturbances at baseline, after short-term treatment had 21 supraventricular episodes (SEs) that spontaneously decreased to two after 18-month PEG (top, left). SE duration also spontaneously reduced after long-term therapy (top, right). On the other hand, after long-term PEG treatment, four sinus pauses (bottom, left) with duration of $200 \mathrm{~ms}$ (bottom, right) were recorded. Bottom panels, case no. 2. At baseline, one patient showed 36 sinus pauses (top), six SEs (centre), and one ectopic beat (bottom). After long-term treatment with PEG, no pauses, no SE, and no ectopic beats were recorded.

cardiac arrhythmias, as well as to the SA-induced relatively scant IGF1 levels, decrease cannot be excluded, although it seems to be unlikely considering that even the normalization of IGF1 induced by SA treatment has been described to exert only a slight but not significant change in the prevalence of arrhythmias in patients with acromegaly $(10,18)$.

SA have been reported to normalize IGF1 levels in up to $60 \%$ of patients and to significantly improve hyperkinetic syndrome in acromegaly $(13,15,22)$. Particularly, clinical and biochemical control of acromegaly induced by medical therapy with SA has been demonstrated to decrease heart rate more significantly than surgery in patients first-line treated with SA (18), suggesting that SA could directly act on cardiomyocytes and pacemaker cells via somatostatin receptors, which are known to be abundantly expressed in the human heart (23). However, SA are listed among the drugs able to prolong the QT
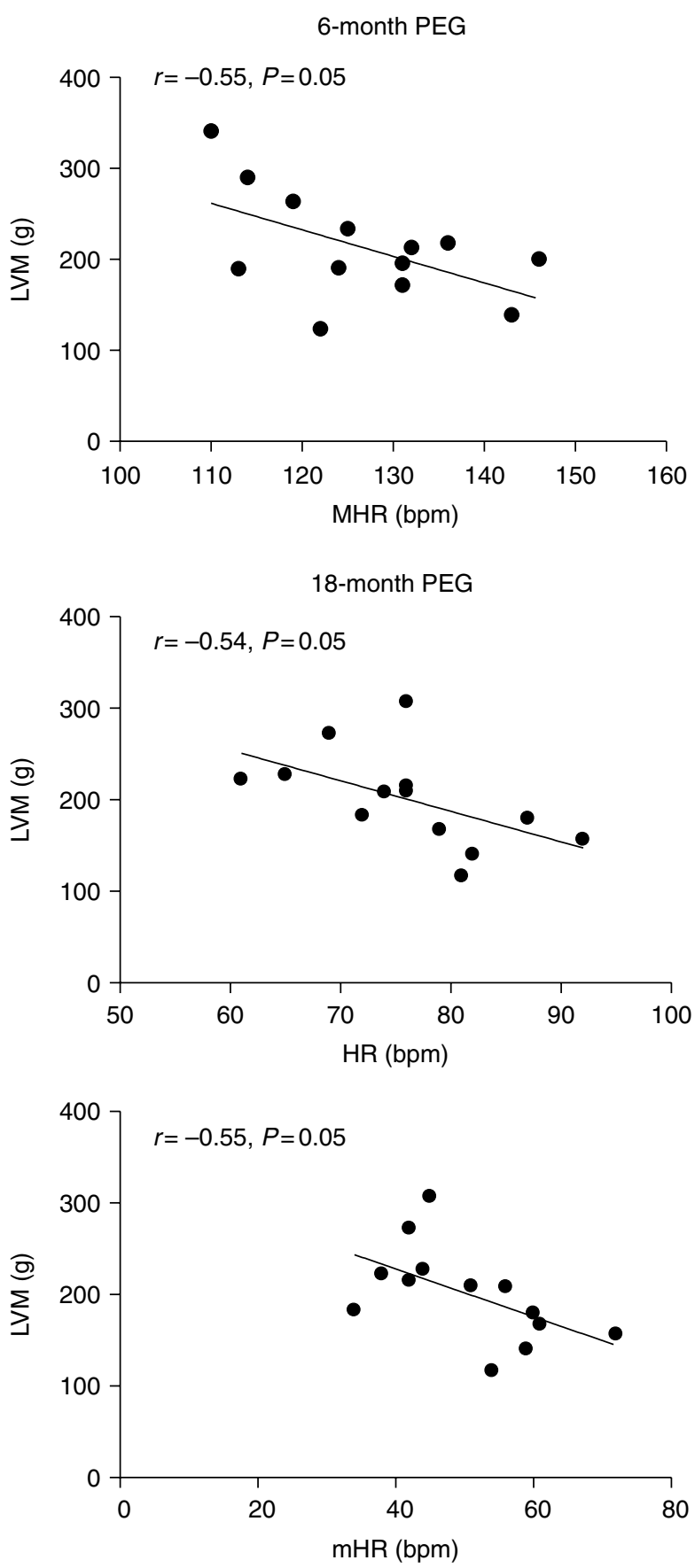

Figure 3 Correlation between LVM and heart rate. After short-term treatment with PEG, LVM was significantly related to MHR (top panel), whereas after long-term treatment LVM was significantly related to HR (centre) and $\mathrm{mHR}$ (bottom). 
interval (24), as native somatostatin has been shown to prolong the effective refractory period of the right ventricle in humans (25).

PEG has been demonstrated to normalize IGF1 levels in up to $90 \%$ of acromegalic patients (26) and in nearly $80 \%$ of those displaying resistance to SA (20). Control of acromegaly induced by PEG has been associated with an improvement of glycometabolic profile and cardiomyopathy in terms of decrease in cardiac mass and increase in ejection fraction (19), in order to enhance cardiac performance and to prevent cardiac failure. This study confirmed that PEG induces a significant improvement in hypertrophic cardiomyopathy and demonstrated that after treatment with PEG, LVM significantly correlated with MHR after short-term treatment and with HR and mHR after long-term treatment, suggesting that PEG might exert a beneficial effect on rhythm disorders and hyperkinetic syndrome, known to be the primum movens of cardiomyopathy in acromegaly.

The role of hypertrophic cardiomyopathy and myocardial fibrosis as key factors in the pathogenesis of rhythm disorders, including malignant ventricular arrhythmias and sudden death, has been extensively investigated and elucidated $(27,28,29,30,31,32,33)$. Heart rate is reportedly inversely associated with LVM (27). Myocardial fibrosis has been demonstrated to be present in $96 \%$ of nonacromegalic patients with arrhythmias and hypertrophic cardiomyopathy $(28,29,30,31,32,33)$ and is implicated as an important substrate in the genesis of life-threatening arrhythmias. In active acromegaly, the underlying structural changes of the heart, most likely due to GH and IGF1 excess on cardiac walls, are myocardial hypertrophy, most invariably present, and myocardial fibrosis found in $85 \%$ of cases in the largest autoptic series (29). Therefore, it is likely that hypertrophic cardiomyopathy and myocardial fibrosis are responsible for the rhythm abnormalities in acromegalics as well. Consequently, the improvement of these cardiac abnormalities, where possible, could induce improvement of arrhythmias. In searching early markers of disturbed cardiac function in active acromegaly, Herrmann et al. (34) have focused on the occurrence of ventricular late potentials and have showed that as many as $56 \%$ of patients with active acromegaly had late potentials on ECG compared with $6 \%$ of patients with well-controlled acromegaly after SA treatment and none of controls. In our patient population, taking into account the long average disease duration ( $>10$ years), the mean age $>40$ years, the high levels of IGF1 at study entry, the presence of hypertrophic cardiomyopathy in $100 \%$ of patients, and the failure of previous long-term high-dose treatment with SA in normalizing IGF1 excess and controlling systemic complications, the presence of rhythm abnormalities does not surprise. It is noteworthy that, despite previous literature reporting that recovery from acromegaly does not seem to significantly improve the rate of conduction disorders $(10,18)$, the results of the current study demonstrated that IGF1 normalization was associated with a reduction by $50 \%$ in the prevalence of arrhythmias, and in one patient, the complete disappearance of rhythm disorders was observed after 18-month PEG. Moreover, this study is the first to document a significant decrease in HR, mHR, and MHR after 18-month PEG. The mechanism underlying this positive effect of PEG treatment on cardiac rhythm in acromegalic patients has not been completely clarified. LVM reduction is likely mediated by normalization of IGF 1 levels, and in turn the significant decrease in LVM after 18-month PEG could significantly impact the prevalence of arrhythmias. However, PEG might exert a beneficial effect on rhythm abnormalities most likely via a direct action on pacemaker cells and on membrane calcium channels, by binding and blocking $\mathrm{GH}$ receptors expressed in the cardiac conduction system. It is noteworthy that in a patient of the current study, a dramatic increase in SEs was recorded after short-term therapy with PEG and spontaneously decreased after long-term PEG treatment. The reasons of this unexpected worsening are still unclear, although in this patient IGF1 and LVM were only slightly reduced after 6-month PEG and normalized only after 18-month PEG. Interestingly, in the patient population of the current study, despite a significant reduction of LVM after shortterm treatment, a significant decrease in HR, mHR, and MHR was observed only after 18-month PEG. Therefore, it can be hypothesized that the decrease in IGF1 levels obtained with PEG treatment is able to rapidly reduce LVM but require more time to improve the heart rate because of a possible relapse in hyperkinetic syndrome occurring after SA discontinuation. This hypothesis seems to be confirmed by the evidence that rhythm parameters are significantly correlated with the change in LVM and the change in IGF1 levels only after longterm therapy with PEG. Alternatively, a long time is necessary before the block of $\mathrm{GH}$ action on cardiac conduction system induces a definite change in heart rate in patients with acromegaly.

In conclusion, the results of this study demonstrate that in acromegalic patients resistant to long-term highdose treatment with SA, PEG treatment induces a significant decrease in HR, MHR, and mHR, suggesting that this treatment may play an important role in the improvement of cardiac arrhythmias associated with acromegaly. Further studies are mandatory to clarify the exact mechanism by which PEG treatment induces this beneficial effect on cardiac rhythm in patients with acromegaly.

\section{Declaration of interest}

The authors declare that there is no conflict of interest that could be perceived as prejudicing the impartiality of the research reported. 


\section{Funding}

This study did not receive any specific grant from any funding agency in the public, commercial, or not-for-profit sector.

\section{References}

1 Melmed S. Acromegaly. New England Journal of Medicine 2006355 2558-2573. (doi:10.1056/NEJMra062453)

2 Colao A, Ferone D, Marzullo P \& Lombardi G. Systemic complications of acromegaly: epidemiology, pathogenesis, and management. Endocrine Reviews 200425 102-152. (doi:10.1210/ er.2002-0022)

3 Nabarro JDN. Acromegaly. Clinical Endocrinology 198726 481-512. (doi:10.1111/j.1365-2265.1987.tb00805.x)

4 Wright AD, Hill DM, Lowy C \& Russell-Fraser T. Mortality in acromegaly. Quarterly Journal of Medicine 1970153 1-16.

5 Bengtsson B, Eden S, Ernest I, Oden A \& Sjogren B. Epidemiology and long-term survival in acromegaly. Acta Medica Scandinavica 1988223 327-335. (doi:10.1111/j.0954-6820. 1988.tb15881.x)

6 Rajasoorya C, Holdaway IM, Wrightson P, Scott DJ \& Ibbertson HK. Determinants of clinical outcome and survival in acromegaly. Clinical Endocrinology 199441 95-102. (doi:10.1111/j.13652265.1994.tb03789.x)

7 Orme SM, McNally RJQ, Cartwright RA \& Belchetz PE. Mortality and cancer incidence in acromegaly: a retrospective cohort study. Journal of Clinical Endocrinology and Metabolism $1998 \mathbf{8 3}$ 2730-2734. (doi:10.1210/jc.83.8.2730)

8 Thuesen L, Christensen SE, Weeke J, Orskov H \& Henningsen P. A hyperkinetic heart in uncomplicated active acromegaly. Acto Medica Scandinavica 1988223 337-343. (doi:10.1111/j.09546820.1988.tb15882.x)

9 Kahaly G, Olshausen KV, Mohr-Kahaly S, Erbel R, Boor S, Beyer J \& Meyer J. Arrhythmias profile in acromegaly. European Heart Journal 199213 51-56.

10 Rodrigues EA, Caruana MP, Lahiri A, Nabarro JD, Jacobs HS \& Raftery EB. Subclinical cardiac dysfunction in acromegaly: evidence for a specific disease of heart muscle. British Heart Journal 198962 185-194. (doi:10.1136/hrt.62.3.185)

11 Colao A, Pivonello R, Grasso LFS, Auriemma RS, Galdiero M, Savastano S \& Lombardi G. Determinants of cardiac disease in newly diagnosed patients with acromegaly: results of a 10 year survey study. European Journal of Endocrinology $2011 \mathbf{1 6 5}$ 713-721. (doi:10.1530/EJE-11-0408)

12 Clayton RN. Cardiovascular function in acromegaly. Endocrine Reviews 200324 272-277. (doi:10.1210/er.2003-0009)

13 Fatti LM, Scacchi M, Lavezzi E, Pecori Giraldi F, De Martin M, Toja P, Michailidist G, Stramba-Badiale M \& Cavagnini F. Effects of treatment with somatostatin analogues on QT interval duration in acromegalic patients. Clinical Endocrinology 200665 626-630. (doi:10.1111/j.1365-2265.2006.02639.x)

14 Montomoli C, Monti MC, Stramba-Badiale M, Marinoni A, Foglieni N, Carreri V, Amigoni M \& Schwartz PJ. Mortality due to sudden infant death syndrome in Northern Italy, 1990-2000: a baseline for the assessment of prevention campaigns. Paediatric and Perinatal Epidemiology 200418 336-343. (doi:10.1111/ j.1365-3016.2004.00583.x)

15 Colao A, Marzullo P, Ferone D, Spinelli L, Cuocolo A, Bonaduce D, Salvatore M, Boerlin V, Lancranjan I \& Lombardi G. Cardiovascular effects of depot long-acting somatostatin analog Sandostatin LAR in acromegaly. Journal of Clinical Endocrinology and Metabolism 200085 3132-3140. (doi:10.1210/jc.85.9.3132)

16 Baldelli R, Ferretti E, Jaffrain-Rea ML, Iacobellis G, Minniti G, Caracciolo B, Moroni C, Cassone R, Gulino A \& Tamburrano G. Cardiac effects of lanreotide, a slow release somatostatin analog in acromegalic patients. Journal of Clinical Endocrinology and Metabolism 199984 527-532. (doi:10.1210/jc.84.2.527)
17 Colao A, Cuocolo A, Marzullo P, Nicolai E, Ferone D, Florimonte L, Salvatore M \& Lombardi G. Effects of one-year treatment with octreotide on cardiac performance in patients with acromegaly. Journal of Clinical Endocrinology and Metabolism 199984 17-23. (doi:10.1210/jc.84.1.17)

18 Colao A, Pivonello R, Galderisi M, Cappabianca P, Auriemma RS, Galdiero M, Cavallo LM, Esposito F \& Lombardi G. Impact of treating acromegaly first with surgery or somatostatin analogs on cardiomyopathy. Journal of Clinical Endocrinology and Metabolism 200823 2639-2646. (doi:10.1210/jc.2008-0299)

19 Pivonello R, Galderisi M, Auriemma RS, De Martino MC, Galdiero M, Ciccarelli A, D’Errico A, Kuorides I, Burman P, Lombardi $\mathrm{G}$ et al. Treatment with growth hormone receptor antagonist in acromegaly: effect on cardiac structure and performance. Journal of Clinical Endocrinology and Metabolism 200792 476-482. (doi:10.1210/jc.2006-1587)

20 Colao A, Pivonello R, Auriemma RS, De Martino MC, Bidlingmaier M, Briganti F, Tortora F, Burman P, Kourides IA, Strasburger CJ et al. Efficacy of 12-months of treatment with the $\mathrm{GH}$ receptor antagonist pagvisomant in patients with acromegaly resistant to long-term, high-dose somatostatin analog treatment: effect on IGF-I levels, tumor mass, hypertension and glucose tolerance. European Journal of Endocrinology 2006154 467-477. (doi:10.1530/eje.1.02112)

21 Foppa M, Duncan BB \& Rohde LEP. Echocardiography-based left ventricular mass estimation. How should we define hypertrophy? Cardiovascular Ultrasound 20053 17. (doi:10.1186/14767120-3-17)

22 Lombardi G, Colao A, Marzullo P, Biondi B, Palmieri E, Fazio S \& the Multicenter Italian Study Group on Lanreotide. Improvement of left ventricular hypertrophy and arrhythmias after lanreotideindiced GH and IGF-I decrease in acromegaly. A prospective multicenter study. Journal of Endocrinological Investigation 200225 971-976.

23 Smith WH, Nair RU, Adamson D, Kearney MT, Ball SG \& Balmforth AJ. Somatostatin receptor subtypes expression in the human heart: differential expression by myocytes and fibroblasts. Journal of Endocrinology 2005187 379-386. (doi:10.1677/joe.1. 06082)

24 The University of Arizona Health Science Center. Center for Education and Research on Therapeutics. Drugs to be avoided by congenital long QT patients. 2002 www.QTdrugs.org.

25 Bubinski R, KUs W \& Goch J. Effects of somatostatin on conduction system of the heart. Kardiologia Polska 199338 258-262.

26 van der Lely AJ, Hutson RK, Trainer PJ, Besser GM, Barkan AL, Katznelson L, Klibanski A, Herman-Bonert V, Melmed S, Vance ML et al. Long term treatment of acromegaly with pegvisomant, a growth hormone antagonist. Lancet 2001358 1754-1759. (doi:10.1016/S0140-6736(01)06844-1)

27 Gidding SS, Carnethon MR, Daniels S, Liu K, Jacobs DR, Sidney S \& Gardin J. Low cardiovascular risk is associated with favourable left ventricular mass, left ventricular relative wall thickness, and left atrial size: the CARDIA study. Journal of American Society of Echocardiography 201023 816-822. (doi:10.1016/j.echo. 2010.05.023)

28 Shiozaki AA, Senra T, Arteaga E, Pita CG, Martinelli Filho M, Avila LF, Parga Filho JR, Mady C \& Rochitte CE. Myocardial fibrosis in patients with hypertrophic cardiomyopathy and high risk for sudden death. Arquivos Brasileiros de Cardiologia 201094 502-506. (doi:10.1590/S0066-782X2010005000017)

29 Lie JT \& Grossman SJ. Pathology of the heart in acromegaly: anatomic findings in 27 autopsied patients. American Heart Journal 19801 41-52. (doi:10.1016/0002-8703(80)90277-X)

30 Courville C \& Mason VR. The heart in acromegaly. Archives of Internal Medicine 193861 704-713. (doi:10.1001/archinte. 1938.00180100014002)

31 Hejtmancik MR, Bradfield JY \& Herrmann GR. Acromegaly and heart: a clinical and pathologic study. Annals of Internal Medicine 195034 1445-1456. 
32 Goldberg MB \& Lisser H. Acromegaly: a consideration of its course and treatment. Report of four cases with autopsies. Journal of Clinical Endocrinology and Metabolism 19422 477-501. (doi:10.1210/jcem-2-8-477)

33 Bogazzi F, Di Bello V, Palagi C, Delle Donne MG, Di Cori A, Gavioli S, Talini E, Cosci C, Sardella C, Brogioni S et al. Improvement of intrinsic myocardial contractility and cardiac fibrosis degree in acromegalic patients treated with somatostatin analogues: a prospective study. Clinical Endocrinology 200562 590-596. (doi:10.1111/j.1365-2265.2005.02265.x)
34 Herrmann BL, Bruch C, Saller B, Ferdin S, Dagres N, Ose C, Erbel R \& Mann K. Occurrence of ventricular late potentials in patients with active acromegaly. Clinical Endocrinology 200155 201-207. (doi:10.1046/j.1365-2265.2001.01319.x)

Received 12 July 2012

Revised version received 17 September 2012

Accepted 12 October 2012 